\title{
Recovery of function after brain damage: Different processes and the facilitation of one
}

\author{
N. DAVIS and T. E. LeVERE \\ North Carolina State University, Raleigh, North Carolina 27650
}

\begin{abstract}
The research reported here was concerned with the role of learning in recovery of function after brain damage. Three experiments were carried out. All of the experiments involved hooded rats trained in a two-choice brightness discrimination when treated with the RNA antimetabolite 8-azaguanine. In normal rats, this drug severely interferes with the acquisition of learned behaviors but not the performance of previously learned behaviors. The first experiment of the present series tested whether this finding was also true for rats required to learn a brightness discrimination without their visual neocortices. The results replicated what was found with normal rats-acquisition was impaired but performance was not. The second experiment investigated the effects of 8-azaguanine on the recovery of a preoperatively acquired brightness discrimination following posterior decortication. The prediction was that if learning was involved in the recovery process, then recovery under the influence of 8-azaguanine should be impaired. However, contrary to this prediction, the recovery process was actually facilitated when the animals were treated with 8-azaguanine. The third experiment tested whether the facilitation found in Experiment 2 was some general facilitation of recovery of function or specifically related to the disruption of learning by 8-azaguanine. The procedure involved testing for recovery of a preopoperative brightness discrimination under conditions where it was reasonable to conclude that the recovery process was a relearning process. In this situation, the drug 8-azaguanine impaired recovery of function. On the basis of these data, we suggest that postoperative learning can interfere retroactively with the reinstatement of spared neural mechanisms when these neural mechanisms are effective in the recovery process.
\end{abstract}

It is well established that neocortical insult can disrupt learned behaviors. One of the better known examples of this is Lashley's (1935) investigation of the effects of visual neocortical ablation. Procedurally, rats were initially trained to discriminate between two brightnesses and then subjected to posterior neodecortication. As a result of this injury, the animals' performance on the brightness discrimination fell to chance. However, the rats were able to reinstate the preoperative behavior if they were retrained on the discrimination, and were able to do so in approximately the same number of trials as required to initially learn the brightness discrimination. This general result has been replicated numerous times (Horel, Bettinger, Royce, \& Meyer, 1966; Meyer, Yutzey, Dalby, \& Meyer, 1968; LeVere \& Morlock, 1973, 1974; LeVere \& Davis, 1977) so that it is pres-

This research was supported by Research Grant NS-12459 from the National Institute of Neurological and Communicative Disorders and Stroke, U.S. Department of Health, Education and Welfare, to T. E. LeVere. The authors wish to thank P. Knowles for her assistance in the preparation of this report. Requests for reprints should be directed to T. E. LeVere, Neuropsychology Laboratory, Department of Psychology, North Carolina State University, Raleigh, North Carolina 27650. ently accepted as one of the more reliable examples of recovery of function.

Perhaps central to this recovery is the fact that certain major components of the disrupted brightness discrimination are spared by the visual ablation. For example, LeVere and Morlock $(1973,1974)$ showed that the neural traces of a preoperatively learned brightness discrimination survive visual decortication by demonstrating that the preoperative learning will proactively interfere with postoperative reversal discrimination training.

More recently, LeVere, Gonder, and Davis (Note 1) have shown that the neural mechanisms associated with the motivation to perform a preoperatively learned brightness discrimination also survive visual decortication. However, notwithstanding this sparing, the lesioned animal still shows an initial behavioral deficit and it becomes important to determine the manner in which appropriate postoperative behavior is reinstated. That is, how recovery of function occurs.

Unfortunately, this determination is severely hampered by an overly general definition of what is to be determined. Traditionally, recovery of function has been taken to indicate an end state which occurs when the brain-injured animal is able to attain the 
same goals postoperatively as were attained preoperatively. Since it is possible to attain goals in any number of ways, the occurrence of recovery of function defined in this manner provides very little appreciation of how recovery occurred. And moreover, recovery of function is not really an end state but rather a process which bridges the gap between an initial behavioral deficit and subsequent effective behavior. The important issue then is not that similar goals may be obtained by the normal and the lesioned animal, but rather how the lesioned animal attains the ability which enables it to do so. For example, since the lesioned animal is initially unable to perform certain learned behaviors, it might be postulated that the recovery process is one of relearning and not dissimilar to that which occurred when the behavior was originally established. On the other hand, given the postoperative sparing of preoperatively established neural traces, the recovery process might be more akin to remembering what was done rather than relearning what to do. The present research is an attempt to dissociate these two fundamental possibilities.

To accomplish this dissociation required that we selectively interfere with either learning or remembering. To meet this requirement, we took advantage of the behavioral effects of the RNA antimetabolite 8-azaguanine. Earlier research with this drug in maze learning tasks (Dingman \& Sporn, 1961) as well as two-choice brightness discrimination tasks (LeVere \& Fontaine, 1978) has demonstrated that the drug will impair acquisition but has no effect on remembering how to perform the tasks once they are learned. With respect to recovery of function, then, one would predict that if recovery is a process where the braininjured animals learns, or relearns, effective behavior patterns, then the postoperative administration of 8-azaguanine should impair the process. On the other hand, if recovery of function is a process of reaccessing spared neural traces and mechanisms as suggested by Meyer (1972), i.e., more like remembering, then the drug should have little effect on how recovery of function progresses.

\section{EXPERIMENT 1}

Unfortunately, the experiments of both Dingman and Sporn (1961) and LeVere and Fontaine (1978) involved intact animals to test the behavioral effects of 8-azaguanine. Since we wished to utilize the drug subsequent to neocortical injury, we were first obligated to demonstrate that the behavioral effects of the drug were not dependent upon the integrity of the neocortex. To accomplish this, we systematically replicated the LeVere and Fontaine experiment, but with animals deprived of their visual neocortices.

\section{Method}

Subjects. Sixteen male hooded rats, between the ages of 90 and 100 days at the time of surgery, were used for this experiment. All animals were housed individually in standard wire mesh cages in a colony which maintained a 10:14-h reversed day/night cycle. Throughout the experiment, the subjects were allowed free access to food and water.

Apparatus. The training apparatus was our automated Y-maze previously described in detail by LeVere and Fontaine (1978). Basically, this apparatus is an equilateral Y-maze with $28-\mathrm{cm}$ arms and a floor composed of brass rods connected to a scrambled shock source. At the end of each arm is a translucent panel which can be illuminated from behind with a 7.5-W lightbulb to produce a luminance of $45-\mathrm{fL}$ at the entrance to the arm. When the translucent panel is not illuminated, the luminance is $.06 \mathrm{fL}$ from the same location. For all training, a lighted rear panel signifies the arm of the maze where the animal may escape footshock and only one arm is safe at any one time.

Procedure. Procedurally, the present experiment was a replication of the LeVere and Fontaine (1978) experiment except for two things. First, there were only two groups, a 6-mg drug group and a saline control group. We employed only a single dosage because we had previously established a dose dependency using the procedures of the present experiment (LeVere \& Fontaine, 1978). Second, all animals were subjected to posterior neodecortication 2 weeks prior to any training experience in the apparatus. This surgery was performed under clean procedures and used sodium pentobarbital as an anesthetic. The lesion was intended to include all neocortex posterior to a line beginning at bregma and becoming tangential to the rhinal sulcus. It is our standard practice to employe lesions of this extent when studying recovery of visual behaviors because we wish to include all neocortex receiving visual projections from the thalamus (Hughes, 1977). Immediately after surgery, each animal was given an injection of a broadband antibiotic as a prophylactic.

Two weeks subsequent to the visual lesion, each animal was shaped to escape footshock in the automated apparatus. On the next day, the animals were trained on the brightness discrimination with the illuminated rear panel signifying the arm where the animal could escape footshock. A training trial began when the rear panel of one arm was illuminated. At the same time, a pulsating footshock (.1-sec pulses) was initiated. This pulsating foot shock was initially at a rate of 1 pulse every $2 \mathrm{sec}$, but systematically increased in frequency over a 1 -min period until it became steady or until the animal had entered the correct arm of the Y-maze. After the animal entered the correct arm, there was a 30-sec intertrial interval before the rear panel of one of the other two arms was illuminated and the next trial started. Intertrial responding was prevented by the occurrence of steady footshock if the animal left the safe arm of the Y-maze. Training continued in this manner with the position of the correct arm varying in a pseudorandom fashion to equate for the number of right and left turns as well as the number of times any particular arm was correct within a 20-trial sequence. Criterion for learning was 9 first-choice correct responses within a series of 10 trials, and each animal was trained to this criterion within a single training session. After criterion performance, each animal was allowed to rest for a 2-day period and then returned to the apparatus and retrained in an identical manner to test for retention.

To determine whether the behavioral effects of 8-azaguanine were dependent on the integrity of visual neocortex, the animals were randomly assigned to two experimental groups. One of these groups $(n=8)$ received a subcutaneous injection of $6 \mathrm{mg}$ of 8 -azaguanine dissolved in $.2 \mathrm{ml}$ of saline $45 \mathrm{~min}$ prior to their initial discrimination training. During the subsequent test for retention, this group was injected with $.2 \mathrm{ml}$ of saline. The second group $(n=8)$ was the mirror image of the first group and received an injection of saline prior to their initial training and an injection of $6 \mathrm{mg}$ of 8 -azaguanine prior to their test for retention. 
If the effects of 8-azaguanine are independent of the integrity of the visual cortex, then one would, of course, predict that the animals injected with the drug during the initial training should be significantly impaired but that there should be no difference between the groups during the test for retention.

Following the test for retention, the animals were deeply anesthetized and sacrificed by intracardial perfusion. The brains of the animals were then removed and the extent of the lesion drawn on standard surface diagrams. Following this, the brains were frozen and sectioned at $50 \mu$. Every fourth section through the area of the neocortical lesion and through the dorsal lateral geniculate nucleus was saved and stained with cresyl violet. This tissue was then microscopically examined to determine the depth of the neocortical damage and the degree of retrograde degeneration within the dorsal lateral geniculate nucleus.

\section{Results and Discussion}

The extent of the neocortical destruction and the retrograde degeneration within the dorsal lateral geniculate nucleus are depicted as blackened areas in the diagrams of Figure 1. The upper portion of the figure is for the animal receiving the smallest neocortical lesion ( $36.3 \%$ of the neocortex), while the bot-
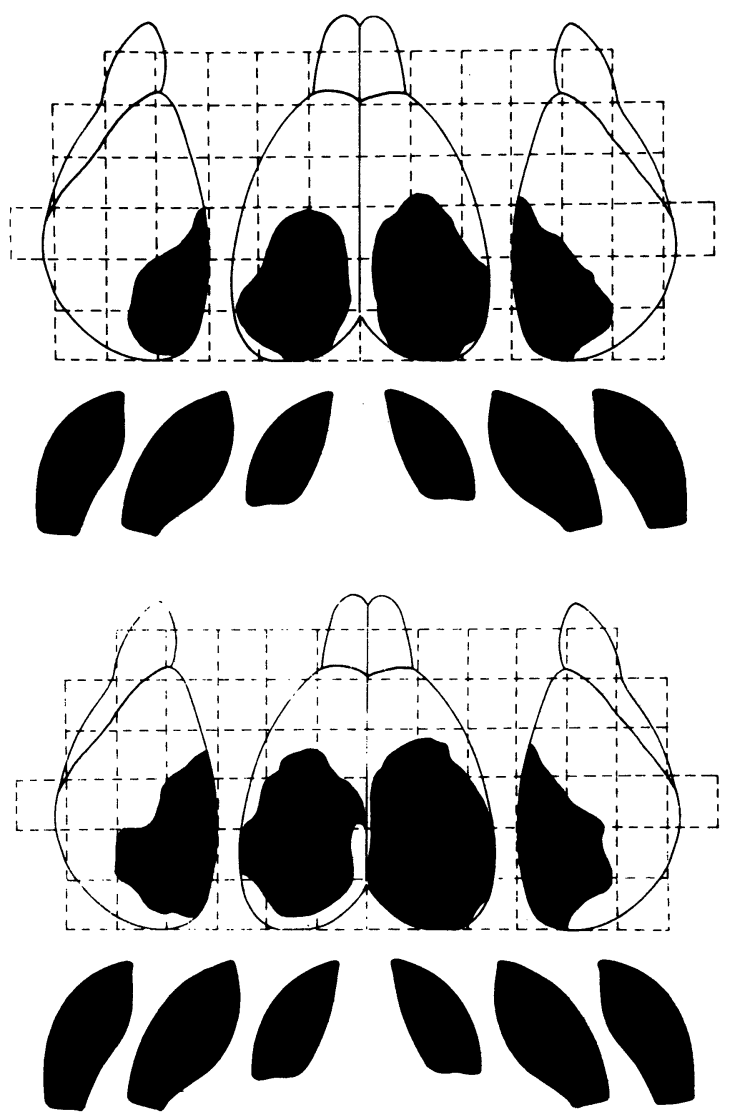

Figure 1. Neocortical destruction and retrograde degeneration within the dorsal lateral geniculate nucleus (black areas) for the rat sustaining the smallest lesion (top) and the rat sustaining the largest lesion (bottom). The three frontal sections through the dorsal lateral geniculate nucleus correspond to $2.5 \mathrm{~mm}$ (extreme left and right), $3.1 \mathrm{~mm}$ (second from left and right), and $3.7 \mathrm{~mm}$ (middle) from the interaural line.

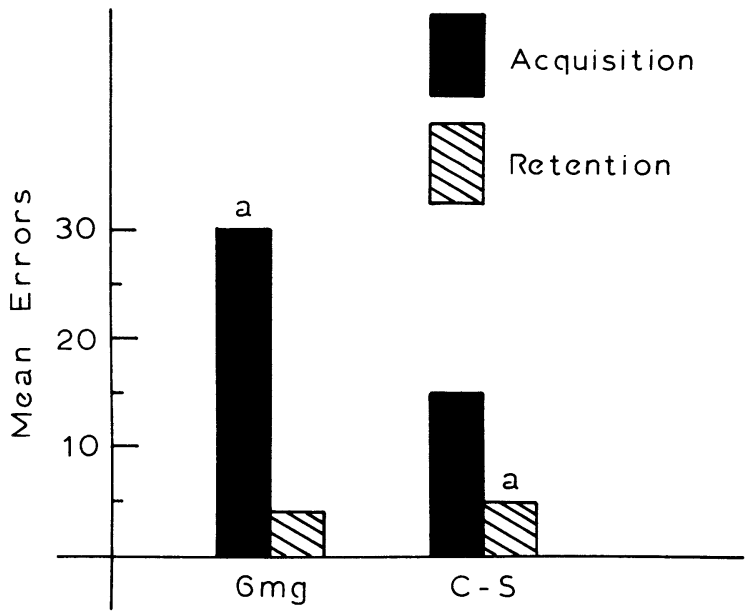

Figure 2. Mean errors to criterion when lesioned rats are initially trained (black bar) and then retrained (cross-hatched bar) 2 days later on a two-choice brightness discrimination. The bars on the left are for the animals initially trained following an injection of 8-azaguanine (a) and then retrained following an injection of saline. The bars on the right are for the animals initially trained following an injection of saline (C-S) and then retrained following an injection of 8-azaguanine (a).

tom portion is for the animal receiving the largest neocortical lesion (40.0\%). Grouping the animals on the basis of whether or not they were initially trained following an injection of $6 \mathrm{mg}$ of 8-azaguanine or an injection of saline, the mean amount of neocortical destruction was, respectively, $42.9 \%$ and $43.7 \%$. This $.8 \%$ difference between the groups was nonsignificant $(t<1)$. The cresyl-violet-stained sections showed that in all cases there was complete destruction of all six neocortical layers. While there was an expected loss of the corpus callosum underlying the lesion, there was never bilateral invasion of the hippocampus or thalamic nuclei. Within the dorsal lateral geniculate nucleus, there was complete retrograde degeneration. Other thalamic nuclei appeared essentially normal, with the exception that the boundary between the dorsal lateral geniculate nucleus and the lateral posterior thalamic nucleus was somewhat difficult to distinguish. However, the boundary between the dorsal and ventral lateral geniculate nuclei was easily visualized and there was no evidence of degeneration within the ventral lateral geniculate nucleus.

The behavioral data of this experiment are presented in Figure 2. This figure shows the mean errors to criterion during the initial training and during the test for retention 2 days later. There was, however, a computer failure during the retention test of one of the animals initially trained after an injection of saline so that the retention data are based on seven animals injected with 8-azaguanine and eight animals injected with physiological saline. As can be seen from the figure, the animals treated with $6 \mathrm{mg}$ of 
8-azaguanine during their initial learning session were significantly impaired relative to the animals injected with just saline $(\mathrm{t}=2.223, \mathrm{df}=14, \mathrm{p}<.05$, two-tailed). However, during retention tests, some 2 days later, there was absolutely no difference between those animals receiving an injection of physiological saline and those animals receiving an injection of 8-azaguanine $(t<1)$.

These data replicate our previous findings with 8 -azaguanine when used with intact rats. In both cases, the drug significantly impairs acquisition but has no effect on the performance of previously learned behaviors. From this, we conclude that the effects of 8 -azaguanine are not dependent upon the integrity of the visual neocortex.

\section{EXPERIMENT 2}

Since 8-azaguanine will interfere with learning, but not performance, in both normal and striate lesioned rats, we believed it would be appropriate to use the drug to investigate the principal issue of the present research. In this respect, it will be remembered that the visual decorticate rat expends a certain number of trials when recovering a preoperatively acquired brightness discrimination. This suggests that postoperative learning or relearning might underwrite the recovery process. On the other hand, it is possible to demonstrate the existence of spared neural traces of the preoperatively acquired brightness discrimination (LeVere \& Morlock, 1973, 1974). And if the neural traces of the preoperatively learned behavior are spared, then why should it be necessary for the lesioned rat to relearn the discrimination? From this point of view, it is possible to suggest that the postoperative recovery process is more like remembering than like relearning.

Our test of these two alternatives was quite straightforward and simply involved the classic Lashley paradigm combined with the postoperative administration of 8-azaguanine. That is, normal rats were trained on a brightness discrimination, subjected to visual decortication, and then required to reinstate the preoperatively acquired brightness discrimination. To determine whether or not learning was involved in this reinstatement, some of the animals were required to recover the preoperative brightness discrimination following an injection of 8-azaguanine. The prediction was that if recovery is a learning process, it should be impaired in those animals treated with 8 -azaguanine. On the other hand, if recovery is a memory process involving the postoperative reinstatement of spared neural traces, it would not depend upon learning and recovery of function should proceed similarly in both the drugged and undrugged animals.

\section{Method}

Subjects. The subjects for this experiment were 19 male hooded rats between the ages of 90 and 100 days. The subjects were maintained as described in Experiment 1.

Apparatus. The apparatus for this experiment was the automated Y-maze described in Experiment 1.

Procedure. The general procedure for this experiment was similar to that of Experiment 1 except for when the animals sustained posterior neodecortication and when the animals were injected with 8-azaguanine. Initially, normal rats were shaped and trained to the $9 / 10$ first-choice correct response criterion in the automated Y-maze within a single day's training session. The following day, each animal sustained posterior neodecortication and was then allowed a 2 -week rest. Following this, the animals were divided into two groups matched on the basis of their preoperative performance and then retrained within a single session to the $9 / 10$ criterion. One of these groups $(n=8)$, however, was given a subcutaneous injection of $6 \mathrm{mg} 8$-azaguanine in $.2 \mathrm{ml}$ of saline $45 \mathrm{~min}$ prior to their retraining. The other group $(n=11)$ was injected with $.2 \mathrm{ml}$ of saline $45 \mathrm{~min}$ prior to their retraining.

The surgery and histological procedures were identical to those described for Experiment 1.

\section{Results and Discussion}

The results of the histological analysis are presented in Figure 3, which is organized identically to that of Figure 1. The extent of the neocortical destruction ranged from $\mathbf{3 5 . 9 \%}$ for the smallest lesion
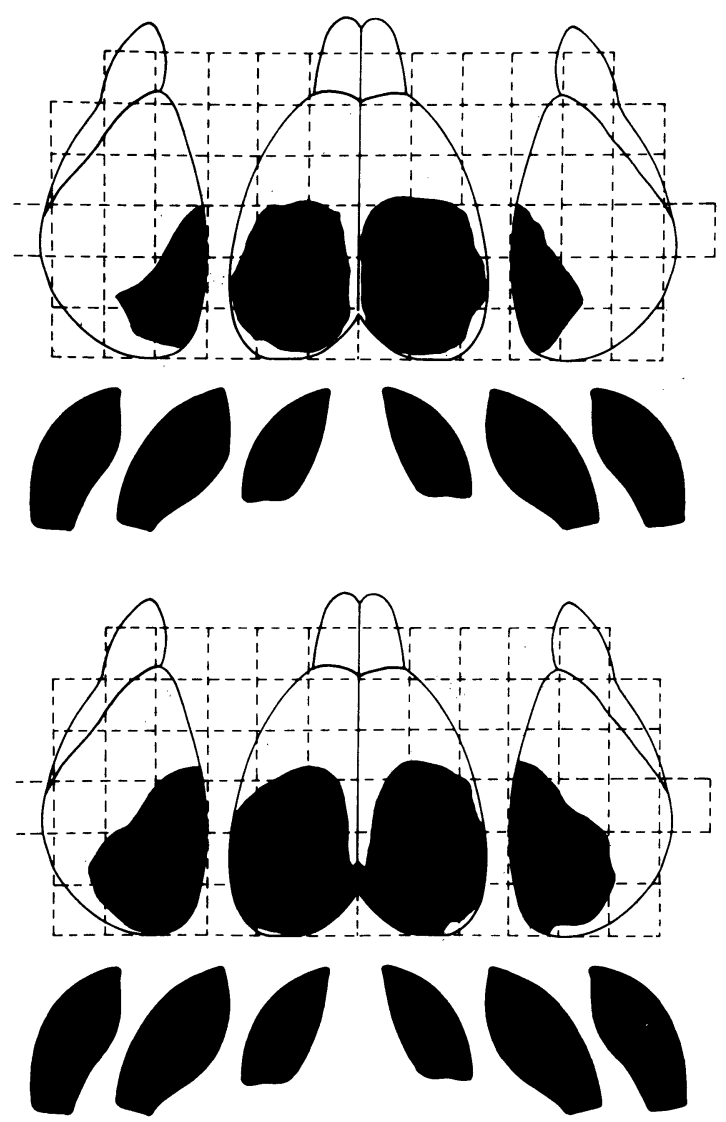

Figure 3. Histological results for animals of Experiment 2. See Figure 1 for explanation. 
to $50.2 \%$ for the largest lesion. The correlation between lesion size and number of errors committed during postoperative retraining was -.28 , which is nonsignificant. The analysis of the cresyl violet stained material yielded results which were identical to those reported in Experiment 1.

The behavioral data are presented in Figure 4. This figure shows the preoperative and postoperative performance of the animals grouped according to whether they were postoperatively retrained following an injection of 8-azaguanine or following an injection of saline. Considering, first, mean errors during preoperative training, there were, of course, no differences between the groups since they were deliberately matched $(t<1)$.

However, as can be seen from the figure, the postoperative performance depended upon whether the animals were retrained following an injection of saline or following an injection of 8-azaguanine. Following an injection of saline, the striate rat commits about the same number of errors postoperatively to reattain criterion as it did when it originally learned the discrimination preoperatively $(\mathrm{t}=1.448, \mathrm{df}=10$, n.s., two-tailed). This, of course, replicates numerous other results, including Lashley's original finding, and simply indicates that an injection of saline has little or no effect on postoperative performance. On the other hand, the postoperative performance of the animals injected with 8-azaguanine was significantly different from their preoperative performance $(\mathrm{t}=6.251, \mathrm{df}=7, \mathrm{p}<.001$, two-tailed $)$. However,

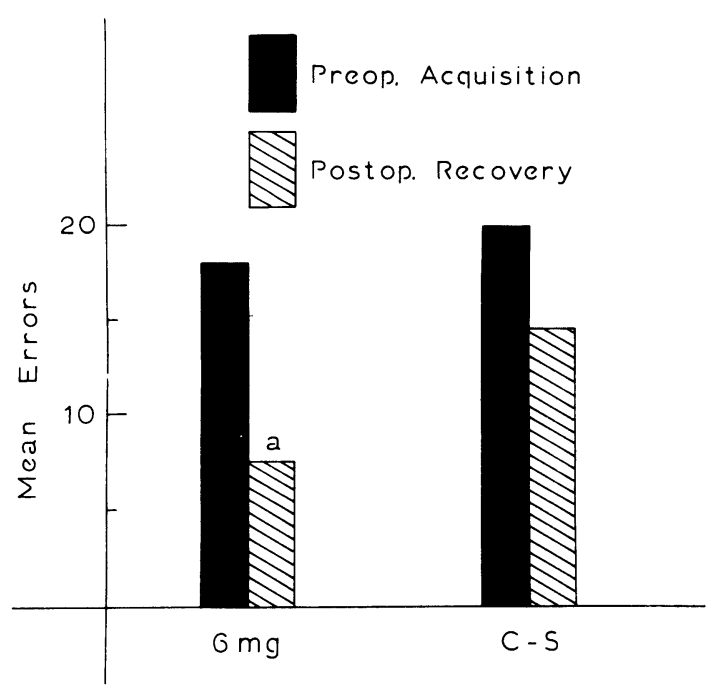

Figure 4. Mean errors to criterion during preoperative initial training (black bars) and postoperative retraining (cross-hatched bars) of a two-choice brightness discrimination. The bars on the left represent the preoperative and postoperative performance of the animals postoperatively retrained following an injection of 8-azaguanine (a), while those on the right are for the animals postoperatively retrained following an injection of saline (C-S). The animals were not injected prior to preoperative initial training. the direction of this difference was quite unexpected. That is, if effective postoperative behavior was mediated through a learning process, then we predicted that the drugged animals would be significantly impaired in their recovery of the brightness discrimination. That 8-azaguanine should facilitate recovery of function was an alternative which we simply had not considered because of the effect this drug has on acquisition and performance in both normal and neocortically injured rats. Yet the drugged animals were clearly superior when recovering the brightness discrimination not only with respect to their own preoperative performance, but also with respect to the postoperative performance of the saline-injected animals $(\mathrm{t}=2.281, \mathrm{df}=17, \mathrm{p}<.05$, two-tailed).

Thus it would seem clear that the striate lesioned rat does not postoperatively relearn a disrupted brightness discrimination. In fact, since 8-azaguanine retards acquisition, it seems reasonable to suggest that the present facilitation occurred because learning may actually impede recovery of function by retroactively interfering with the expression of what is spared by the brain injury.

\section{EXPERIMENT 3}

Before dignifying with further consideration the suggestion that recovery of function may in certain instances be accomplished by a process like remembering and that new learning can interfere with the process, it is necessary to resolve an alternate interpretation. This is that to forward this conclusion on the basis of the previous experiment requires assuming that the drug 8-azaguanine does not have some more direct effect on recovery of function in general and spared neural mechanisms in particular. For example, it is possible to suggest that brain injury increases RNA metabolism and that this disruption of normal brain biochemistry is what leads to a behavioral deficit. Or it is possible to suggest that 8-azaguanine may directly potentiate spared neural mechanisms and thereby mitigate a postoperative behavioral deficit. In either case, the facilitation of recovery of function by 8-azaguanine would be a direct effect of the drug and not a secondary consequence of interfering with new learning.

To test these alternatives required testing 8-azaguanine in a postoperative recovery situation where preventing new learning would not facilitate the expression of spared neural traces or neural mechanisms. Fortunately, it is possible to produce such a situation by changing the motivation between preoperative learning and postoperative recovery of function. When this is done, the spared neural mechanisms and/or neural traces of a preoperatively learned brightness discrimination do not influence postoperative recovery of function (LeVere \& Davis, 1977). 
Since spared neural traces do not influence recovery, the process cannot be facilitated by preventing new learning and thereby reducing retroactive interference. However, if it was a more direct effect of reducing RNA metabolism which facilitated recovery of function in Experiment 2, then 8-azaguanine should similarly facilitate recovering the brightness discrimination, even though retrograde interference is not an issue. On the other hand, if it is, in fact, the more indirect consequence of disrupting new learning and reducing retrograde interference which facilitated recovery of function in Experiment 2, then the postoperative administration of 8-azaguanine should retard the process. This is so because if spared neural traces do not participate in the recovery of the preoperative behavior, then the lesioned animal must, by definition, relearn the discrimination to recover. And if the animal is recovering the behavior through relearning, then anything which disrupts learning should interfere with recovery.

\section{Method}

Subjects. Sixteen male hooded rats between the ages of 90 and 100 days served as subjects. In the present experiment, the animals were initially trained for water reinforcement, and during this time each was $23 \mathrm{~h}$ water deprived prior to training. At all other times, the subjects were allowed free access to food and water.

Apparatus. The initial water-reinforced training of the present procedure required that we use our standard Yerkes brightness discrimination apparatus. This apparatus has been previously described by LeVere and Davis (1977) and is composed of a start compartment, a choice compartment, and two goal compartments which are distinguished by transilluminated entrance doors. The floor of the start and choice compartments is made of brass rods which are connected to a scrambled shock source for shock avoid/ escape training. When the animals are trained for water reward, no shock is administered and both goal compartments are baited with a small quantity of water to eliminate olfactory cues. However, as during shock-avoidance/escape training, only the entrance door to the correct goal compartment is unlocked.

The discriminative cues for the present experiment were produced by flood lamps at the end of enclosed tunnels extending backward from each goal compartment. The flood lamps were powered by an ac voltage necessary to produce a 64 -fL brightness on the entrance doors as measured from within the choice compartment. This brightness served as the brighter cue. The dimmer discriminative cue was produced by interposing a small aperture in front of one of the flood lamps to restrict its light output to $1 \mathrm{fL}$. As in Experiments 1 and 2, all animals were trained with the brighter cue signifying the correct goal compartment.

Procedure. Initially, the 16 subjects were shaped to run in the Yerkes apparatus and enter the goal compartment to obtain water. Following this, the animals were given training on the brightness discrimination at the rate of 10 trials/day for 7 days each week. The training procedure was a correction procedure to allow the animal to correct an initial error and obtain water reinforcement on each trial. The position of the correct goal compartment varied in a pseudorandom fashion so that during each day's training the animal was rewarded equally often in the left and the right goal compartments. Criterion was 9 first-choice correct responses in the 10 trials of a day's training. On the day following reaching this criterion, each animal was subjected to posterior neodecortication following the procedures described in Experiment 1.
Subsequent to surgery, each animal was allowed a 2-week rest period. Following this, the animals were returned to the Yerkes apparatus and retrained on the preoperatively learned brightness discrimination. However, during this postoperative training, the animals were fully satiated and were trained to avoid/escape footshock according to the procedures detailed by LeVere and Davis (1977). Since the animals were administered drugs and since appetitive satiation was not a consideration, all animals were, however, trained to the $9 / 10$ criterion within a single session. During this postoperative training session, nine of the animals received an injection of $6 \mathrm{mg}$ of 8 -azaguanine dissolved in $.2 \mathrm{ml}$ saline $45 \mathrm{~min}$ prior to their training session. The remaining seven animals received an injection of $.2 \mathrm{ml}$ saline $45 \mathrm{~min}$ prior to their postoperative training.

After completing postoperative training, the animals were sacrificed for histological analysis as described in Experiment 1.

\section{Results and Discussion}

The histological results of the present experiment are presented in Figure 5. The upper portion of this figure represents the animal sustaining the smallest amount of neocortical injury $(45 \%)$, while the lower portion is for the animal sustaining the largest neocortical injury $(51 \%)$. Testing for differences in lesion size between the two experimental groups showed no significant differences $(t<1)$. Microscopic analysis of the cresyl-violet-stained material yielded the same results as reported for Experiments 1 and 2.
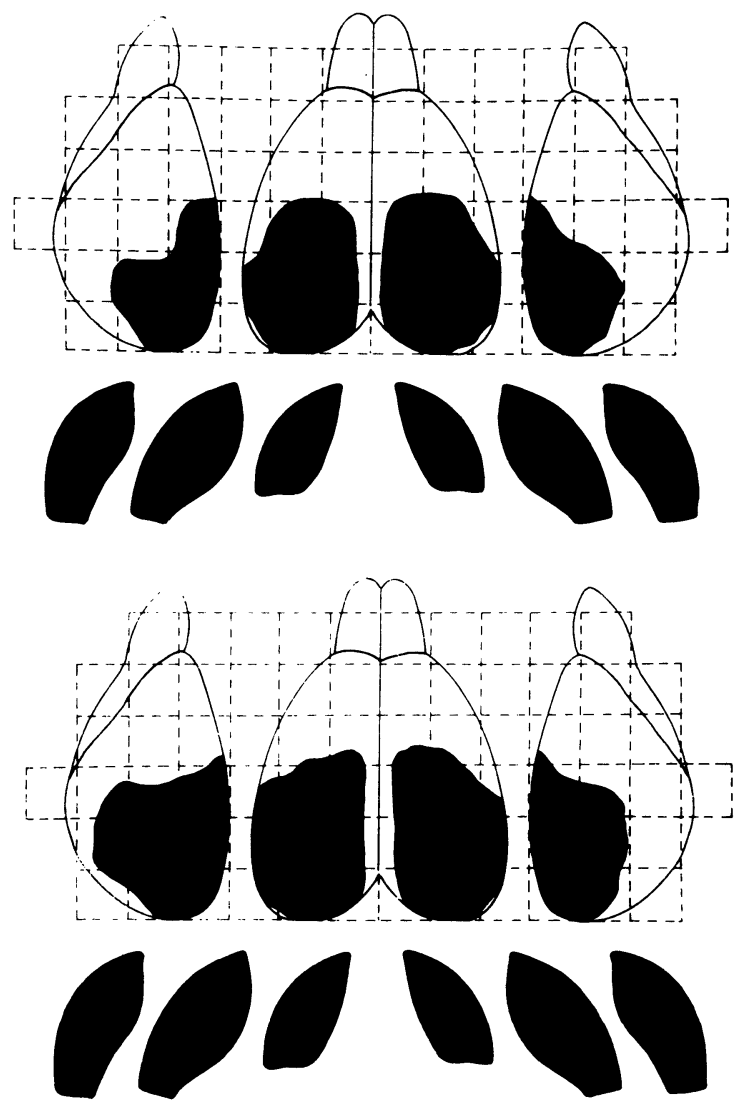

Figure 5. Histological results of Experiment 3. See Figure 1 for explanation. 


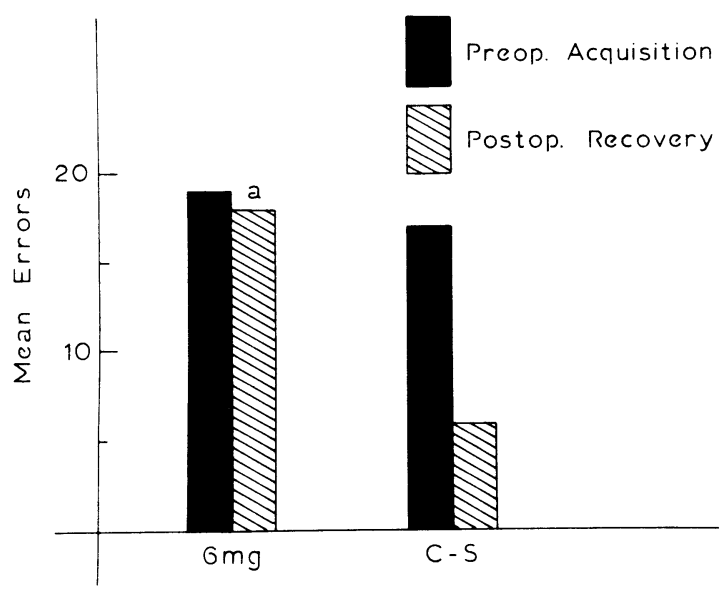

Figure 6. Mean errors to criterion during preoperative initial training (black bars) and postoperative retraining (cross-hatched bars) of a two-choice brightness discrimination. Preoperative training was water reinforced, while postoperative retraining was to avoid/escape footshock. The bars on the left represent the preoperative and postoperative performance of the animals postoperatively retrained following an injection of 8-azaguanine (a), while those on the right are for the animals postoperatively retrained following an injection of saline $(\mathrm{C}-\mathrm{S})$. The animals were not injected prior to preoperative initial training.

The behavioral data are presented in Figure 6, which shows the mean errors to criterion during preoperative learning and postoperative recovery. The figure groups the animals according to whether they were postoperatively retrained following an injection of 8-azaguanine or following an injection of saline. During preoperative training, there were no differences between the mean error scores of the two groups $(t<1)$. However, during postoperative recovery, the animals retrained following an injection of 8-azaguanine committed virtually three times as many errors as the animals retrained following an injection of physiological saline. This is, of course, statistically reliable according to conventional standards $(\mathrm{t}=4.583, \mathrm{df}=14, \mathrm{p}<.001$, two-tailed $)$.

From these data, we conclude that the facilitation of postoperative recovery of function in Experiment 2 was not a function of the disruption of RNA metabolism per se. Rather, we submit that recovery was facilitated in Experiment 2 because RNA metabolism is involved in new learning, and when this is prevented, the animal is better able to, perhaps forced to, more quickly utilize the spared neural mechanisms which eventually will influence recovery of function. Or, put otherwise, recovery of function was facilitated in Experiment 2 because retroactive interference was prevented. However, in the present experiment, the spared neural mechanisms or neural traces do not influence recovery of function because of the change in motivation between preoperative and postoperative training. In this case, recovery is more probably a learning process and, as such, is adversely affected by the RNA antimetabolite 8azaguanine.

\section{DISCUSSION}

Under appropriate conditions, it is possible to demonstrate that neocortical injury does not destroy the neural traces of a preoperatively learned behavior even though the individual is initially unable to perform the behavior (LeVere, 1975; LeVere \& Davis, 1977; LeVere \& Morlock, 1973, 1974). Moreover, not only are these neural traces spared, but they are also able to affect the progress of recovery of function provided there is a close similarity between the preoperative and postoperative situations (LeVere \& Davis, 1977; also see Davis \& LeVere, Note 2). The question of the present series of experiments was whether or not the postoperative effectiveness of these spared neural traces was established through learning or remembering.

To address this question, we used the RNA antimetabolite 8-azaguanine to disrupt new learning. Since this drug does not affect the performance of previously learned behaviors, our prediction was that if the lesioned animal learns to utilize what is spared during postoperative recovery, then the administration of this drug should retard the recovery process. However, not only did the results of our experiments fail to support this prediction, they were, in fact, directly opposite (Experiment 2 ). From this we conclude that the postoperative reinstatement of the behavioral potency of spared neural mechanisms is not a process which involves learning. On the contrary, under conditions when spared neural mechanisms will influence recovery of function, learning actually impairs the recovery process.

This is, of course, not to say that learning is always detrimental to recovery of function after brain injury. It is important to emphasize the qualification that learning can interfere with recovery of function when, perhaps only when, spared neural traces participate in the recovery process. When there are no existing neural traces (Experiment 1) or when the spared neural traces do not participate in the recovery process (Experiment 3), then the only avenue open for recovery is learning or relearning which is obviously not detrimental. In this respect, we believe that the drug had the same single effect in each of the three experiments and that this effect was to disrupt learning. In Experiments 1 and 3, this disruption was to the detriment of the brain-injured rat, because in these experiments the animals were learning a brightness discrimination without the aid of spared preoperatively established neural mechanisms or neural traces. In Experiment 2, however, this same disruption of learning was to the benefit of the lesioned animal, since the animals were recovering a specific 
spared behavior and it served to free the expression of spared neural mechanisms from the effects of retroactive interference.

This then brings us to perhaps the critical message of the present research. That is, defining recovery of function as the reestablishment of behaviors to obtain certain preoperative goals is virtually meaningless with respect to mechanisms of the process. This is so because the method of establishing appropriate postoperative behavior may be quite different even though the end result is the same. The evidence for this is that the same postoperative experimental manipulation can produce diametrically opposed results. And this must necessarily mean that what is occurring postoperatively in one case is quite different from what is occuring in another case. For example, in each experiment of the present series, the postoperative behavioral goal was appropriate discriminatory behavior, and in each experiment the animals attained this goal. However, in Experiment 1, where spared neural traces were not present, or in Experiment 3, where spared neural traces do not influence recovery of function, the animals attained this goal through a learning process. On the other hand, in Experiment 2, where it is possible to demonstrate the postoperative influence of spared neural traces, the postoperative process was more akin to remembering what to do rather than learning what to do. In this regard, the suggestion by Meyer (1972) and by Meyer and Meyer (1977) that the behavioral deficit following brain injury may, in certain instances, represent a memory dysfunction is clearly supported and represents a conclusion of remarkable insight.

\section{REFERENCE NOTES}

1. LeVere, T. E., Gonder, L., \& Davis, N. Recovery of function after brain damage: An evaluation of whether the destruction of motivational systems may be responsible for the visual deficits following striate lesions. Manuscript submitted for publication, 1978.
2. Davis, N., \& LeVere, T. E. Recovery of function after brain damage: An explanation of the nature of the behavioral deficit following neocortical injury. Manuscript submitted for publication, 1978.

\section{REFERENCES}

Dingman, W., \& Sporn, M. B. The incorporation of 8-azaguanine into rat brain RNA and its effect on maze learning by the rat: An inquiry into the biochemical basis of memory. Journal of Psychiatric Research, 1961, 1, 1-11.

Horel, J. A., Bettinger, L. A., Royce, G. J., \& Meyer, D. R. Role of neocortex in the learning and relearning of two visual habits by the rat. Journal of Comparative and Physiological Psychology, 1966, 61, 66-78.

Hughes, H. C. Anatomical and neurobehavioral investigations concerning the thalamo-cortical organization of the rat's visual system. Journal of Comparative Neurology, 1977, 175, 311-335.

Lashley, K. S. The mechanism of vision. XII. Nervous structures concerned in habits based on reactions to light. Comparative Psychology Monographs, 1935, 11, 43-79.

LEVERE, T. E. Neural stability, sparing, and behavioral recovery following brain damage. Psychological Review, 1975, 82, 344-358.

LEVERE, T. E., \& DAvis, N. Recovery of function after brain damage: The motivational specificity of spared neural traces. Experimental Neurology, 1977, 57, 883-899.

LeVere, T. E., \& Fontaine, C. W. A demonstration of the importance of RNA metabolism for the acquisition but not performance of learned behaviors. Experimental Neurology, 1978, 59, 444-449.

LEVere, T. E., \& Morlock, G. W. The nature of visual recovery following posterior decortication in the hooded rat. Journal of Comparative and Physiological Psychology, 1973, 83, 62-67.

LEVERE, T. E., \& MorLock, G. W. The influence of preoperative learning on the recovery of a successive brightness discrimination following posterior neodecortication in the hooded rat. Bulletin of the Psychonomic Society, 1974, 4, 507-509.

Meyer, D. R. Access to engrams. American Psychologist, 1972, 27, 124-133.

Meyer, D. R., \& Meyer, P. M. Dynamics and bases of recoveries of functions after injuries to the cerebral cortex. Physiological Psychology, 1977, 5, 133-165.

Meyer, P. M., Yutzey, D. A., Dalby, D. A., \& Meyer, D. R. Effects of simultaneous septal-visual, septal-anterior and anterior-posterior lesions upon relearning of a black-white discrimination. Brain Research, 1968, 8, 281-290.

(Received for publication January 26, 1979; revision accepted July 9, 1979.) 